OPEN ACCESS

Edited by:

Wan Li,

Harbin Medical University, China

Reviewed by:

Chun Wang,

Thomas Jefferson University,

United States

Xu Guo,

Fourth Military Medical University,

China

*Correspondence:

Shaogui Wan

wansg@gmu.edu.cn

Jingxiang Xu

xunov10@sina.com

Specialty section:

This article was submitted to

Genomic Assay Technology,

a section of the journa

Frontiers in Genetics

Received: 02 June 2021

Accepted: 17 August 2021

Published: 16 September 2021

Citation:

Zhou G, Li Y, Li S, LiU H, XU F, Lai $X$, Zhang $Q, X U$ J and Wan $S$ (2021) Circulating Cell-Free mtDNA Content as a Non-invasive Prognostic Biomarker in HCC Patients Receiving

TACE and Traditional Chinese

Medicine. Front. Genet. 12:719451.

doi: 10.3389/fgene.2021.719451

\section{Circulating Cell-Free mtDNA Content as a Non-invasive Prognostic Biomarker in HCC Patients Receiving TACE and Traditional Chinese Medicine}

\author{
Guanlin Zhou ${ }^{1}$, Ying $\mathrm{Li}^{2}$, Shicheng $\mathrm{Li}^{2}$, Hongxia Liu', Fei Xu${ }^{1}$, Xiaohuan Lai \\ Qiong Zhang ${ }^{3}$, Jingxiang $X u^{2 *}$ and Shaogui Wan ${ }^{1,2 *}$
}

'Institute of Hepatology, Department of Hepatology, The Affiliated Fifth People's Hospital of Ganzhou, Gannan Medical University, Ganzhou, China, ${ }^{2}$ Center for Molecular Pathology, Department of Basic Medicine, Gannan Medical University, Ganzhou, China, ${ }^{3}$ Department of Emergency Medicine, First Affiliated Hospital, Gannan Medical University, Ganzhou, China

Hepatocellular carcinoma (HCC) accounts for $70-85 \%$ of liver cancer, and about $85 \%$ of $\mathrm{HCC}$ are hepatitis B virus-related (HBV-HCC) in China. Transarterial chemoembolization (TACE) combined with traditional Chinese medicine (TCM) has been reported as an effective treatment. Potential biomarkers to stratify patients who may benefit from this treatment are needed. In this study, we aimed to evaluate whether circulating cell-free mitochondrial DNA (ccf-mtDNA) content was associated with the outcome of HCC patients, especially of those who received the combination treatment of TACE and TCM. Univariate and multivariate Cox analyses were conducted to evaluate the association between ccf-mtDNA content and the overall survival of HBV-HCC patients. KaplanMeier analysis was used to compare the survival differences between patients with low and high ccf-mtDNA content. In a hospital-based cohort with 141 HBV-HCC patients, there was no statistically significant association between the ccf-mtDNA content and the overall survival of HBV-HCC patients in the univariate analysis, but a borderline significant association was found in the multivariate analyses. In a subcohort of $50 \mathrm{HBV}$ HCC patients who received TACE and TCM treatment, high ccfDNA content conferred an increased death risk with a hazard ratio of 4.01 (95\% confidence interval: 1.25-12.84, $p=0.019$ ) in the multivariate analysis. Kaplan-Meier survival analysis also showed that patients with high ccf-mtDNA content had unfavorable survival (log rank $p=0.097$ ). Our findings suggest that ccf-mtDNA content is a potential non-invasive prognostic biomarker in HCC patients receiving TACE and TCM treatment.

Keywords: hepatocellular carcinoma, mitochondrial DNA, TACE, non-invasive biomarker, clinical outcome

\section{INTRODUCTION}

Hepatocellular carcinoma (HCC) is the second leading cause of cancer death, and chronic hepatitis B virus (HBV) infection accounts for at least 50\% of HCC cases worldwide (Xie, 2017). Notably, HBV-related HCC accounts for about $85 \%$ of HCC cases in China. The vast majority of HCC patients are diagnosed at middle or later stages, and curative treatments like surgical resection 
are not suitable for these patients (Gomaa, 2015). Transarterial chemoembolization (TACE) is the standard of care for patients with intermediate HCC according to the Barcelona Clinic Liver Cancer staging system. Previous studies reported that TACE combined with traditional Chinese medicine (TCM) regimen displayed a high efficacy in treating advanced HCC (Chen et al., 2019).

The mitochondrion is a ubiquitous eukaryotic cell organelle which plays an important role in energy production, cell proliferation, as well as apoptosis (Veltri et al., 1990). The circular genome of the mitochondria (mtDNA) encodes for proteins essential in the oxidative phosphorylation system and the tRNA and rRNA molecules of the mitochondrial translation apparatus. Mitochondrial dysfunction plays an important role in the occurrence and development of liver cancer (Xiong et al., 2012; Dilip et al., 2013), and when mitochondrial dysfunction causes mtDNA damage, the mtDNA fragments can escape from the matrix and enter the cytosol or systemic circulation (Wenceslau et al., 2014). Changes in mtDNA content of tumor specimens have been reported recently (Reznik et al., 2016). The alteration of mtDNA content or sequence mutations has been involved in carcinogenesis and progression and thus becomes a potential predictive and prognostic biomarker for certain types of cancers (Fliss et al., 2000). Bao et al. (2016) found that the mtDNA copy number was associated with overall survival in HCC patients treated with TACE. mtDNA may also be released at low levels into the circulation from the mitochondria under cellular stress, which results in circulating cell-free mtDNA (ccfmtDNA) being detectable in blood samples (Yu, 2012). Recently, circulating mtDNA was suggested to be a novel non-invasive biomarker for cancer diagnosis and prognostic evaluation due to its specific and unique characteristics (Li et al., 2016; Weerts et al., 2018), for example, the study of Li et al. (2016) showed that the serum ccf-mtDNA content of HBV-HCC patients was significantly lower than that of cancer-free HBV controls, and compared to HBV patients with high mtDNA content, those with low mtDNA content had a significantly increased risk of HCC. However, the association of ccf-mtDNA content with the clinical outcomes of HCC patients remains largely unknown.

In this study, using real-time quantitative PCR method, we measured the ccf-mtDNA content in a hospital-based cohort of HCC patients and further evaluated the association of ccfmtDNA content with the clinical outcomes of HCC patients, especially of those who received TACE and TCM treatment.

\section{MATERIALS AND METHODS}

\section{Patients}

Informed consent was obtained from each patient according to the protocol approved by the ethics committee of the Affiliated Fifth People's Hospital of Ganzhou in Gannan Medical University. A total of $148 \mathrm{HBV}$-HCC patients were enrolled at the Department of Hepatology, Affiliated Fifth People's Hospital of Ganzhou in Gannan Medical University between January 2015 and October 2018. Each patient has two or more serum samples collected at different time points, and the first serum sample (baseline) after the patient was diagnosed with HCC was examined in this study. After excluding seven patients who had been diagnosed with HCC before their admission to this study, the final cohort included 141 patients with HBV-HCC (Figure 1). The statistical analysis was also conducted in a subcohort of 50 patients treated with TACE combined with TCM (Yangzhengxiaoji capsule, $n=23$; Antike capsule, $n=5$; and Huachansu capsule, $n=17$; combination TCM treatments, $n=5$ ).

\section{Serum Sample Preparation}

Whole blood sample from each patient was collected in nonanticoagulated blood collection tubes and then transferred to the laboratory for processing within $6 \mathrm{~h}$. The serum samples were collected by two-step centrifugation: the serum sample was extracted from the whole blood after centrifugation at 1,500 rpm for $5 \mathrm{~min}$, then the supernatant was removed to a clean tube and re-centrifuged with $12,000 \mathrm{rpm}$ for another $5 \mathrm{~min}$, and finally, the supernatant was collected into a $1.5-\mathrm{ml}$ tube and stored at $-80^{\circ} \mathrm{C}$ for use.

\section{Measurement of Serum mtDNA Content}

QIAamp DNA Blood Mini kit (Qiagen, Carlsbad, CA, United States) was used to isolate the circulating DNA from a 200- $\mu$ l serum sample according to the protocol of the manufacturer. mtDNA content was measured by real-time qPCR with modified protocol as described by previous studies (Fu et al., 2012; Wan et al., 2012), in which the ratio of the copy number for mitochondrial ND1 gene to the copy of a human single copy gene 36B4 was used to determine the relative mtDNA content. Briefly, the primer of ND1 gene was used for mtDNA amplification with the primer sequences as follows: ND1-F: 5'-CCCTAAAACCCGCCACATCT-3' and ND1-R: 5'-GAGCGATGGTGAGAGCTAAGGT-3'. The primer of 36B4 gene was used as a single copy gene control in all reactions, and the primer sequences were as follows: 36B4-F: $5^{\prime}$-CAGCAAGTGGGAAGGTGTAATCC-3' and 36B4-R: $5^{\prime}$ CCCATTCTATCATCAACGGGTACAA-3'. The 20- $\mu \mathrm{l}$ qPCR reaction for ND1 and 36B4 consisted of $1 \times$ TB Green fast qPCR Mix $(2 \times)$ (Takara), $0.2 \mu \mathrm{M}$ of each primer, and $2 \mu \mathrm{l}$ of purified serum DNA sample. The thermal cycling conditions were $95^{\circ} \mathrm{C}$ for $10 \mathrm{~min}$, followed by $40 \mathrm{cycles}$ of $95^{\circ} \mathrm{C}$ for $15 \mathrm{~s}$, and $60^{\circ} \mathrm{C}$ for $60 \mathrm{~s}$ with signal acquisition. All samples were assayed in duplicate on a 96-well plate using the QuantStudio ${ }^{\mathrm{TM}} 7$ Flex qRT-PCR system (Applied Biosystems, United States). The same negative control and calibrator DNA samples were incorporated into each plate for quality control and the calibration of PCR efficiency. A reference DNA sample was used to construct a standard curve for mtDNA measurement in each plate. For each standard curve, the reference DNA sample was serially diluted by $1: 3$ to produce a six-point standard curve. The $R^{2}$ for each standard curve was $\geq 0.99$.

\section{Statistical Analysis}

IBM SPSS 20.0 statistical software was used to analyze the collected clinical data and ccf-mtDNA. Continuous variables were represented by average with standard deviation (mean $\pm \mathrm{SD}$ ) or median with interquartile range, according to 


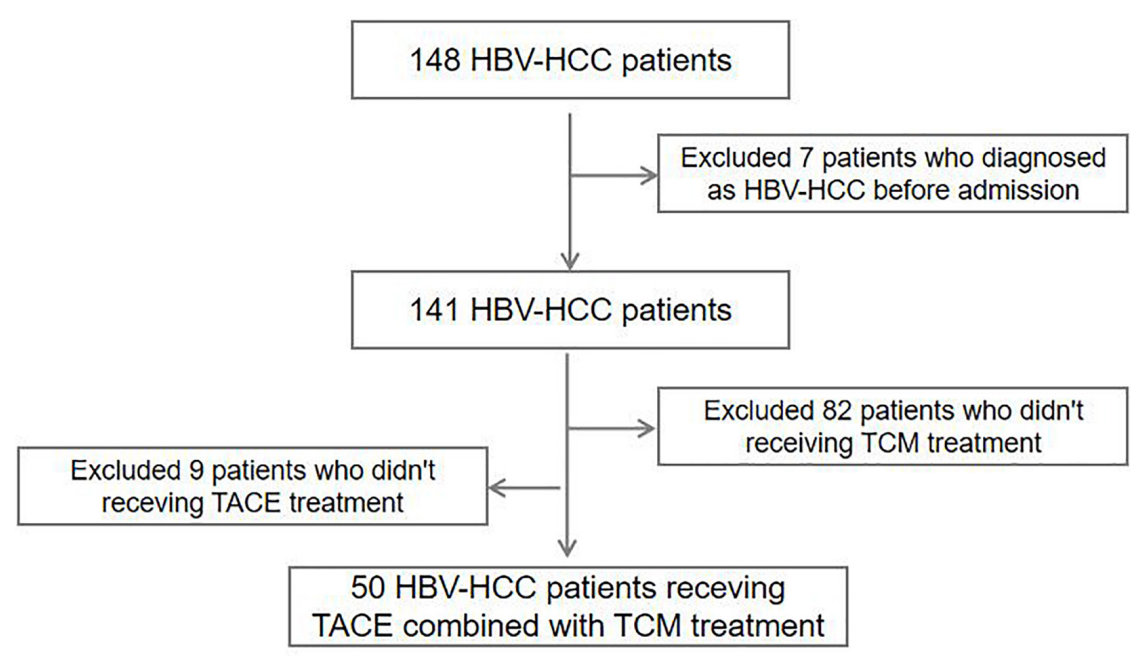

FIGURE 1 | Flowchart of patient enrolment.

the data distributions. Categorical variables were described using frequency and percentage. Cox proportional hazards model was used for the univariate and multivariate analyses. Kaplan-Meier method was used to draw the survival curves, and log-rank test was used for comparisons of survival between groups. Moreover, $p$-value $<0.05$ was considered as the threshold of statistical significance.

\section{RESULTS}

\section{Characteristics of HBV-HCC Patients}

The epidemiological and clinical characteristics of $141 \mathrm{HBV}-\mathrm{HCC}$ patients are summarized in Table 1 . The average age of the study population was 56.96 (SD 11.34) years, and most of the patients were male $(83.7 \%)$, non-smokers $(81.9 \%)$, non-drinkers $(80.9 \%)$, and without a family history of cancer (73\%). There were 45 (31.9\%) patients who developed liver cirrhosis and 117 (83.0\%) patients with a B or C Child-Pugh class. Among these 141 HBV-HCC patients, 70 (49.6\%) patients were treated with TACE, $59(41.8 \%)$ patients were treated with TCM, and $50(35.4 \%)$ patients received a combination therapy of TACE and TCM. During a median follow-up of 28.0 months (interquartile range, 18.0-37.0 months), 88 (62.4\%) patients have died.

\section{Association Between ccf-mtDNA Content and Overall Survival of HBV-HCC Patients}

The median and tertile values of ccf-mtDNA content were used to group patients, and the association between ccf-mtDNA content and the overall survival of HBV-HCC patients was evaluated using univariate and multivariate Cox analyses. As shown in Table 2, there was no statistically significant association between ccf-mtDNA content and the overall survival of HBV-HCC patients in the univariate analysis. However, in the multivariate analyses adjusting for age, gender, drinking and smoking status,
TABLE 1 | Characteristics of the study population.

\begin{tabular}{|c|c|}
\hline Characteristics & Number $(n=141, \%)$ \\
\hline \multicolumn{2}{|l|}{ Age (median) } \\
\hline$\leq 57$ & $71(50.4)$ \\
\hline$>57$ & $70(49.6)$ \\
\hline \multicolumn{2}{|l|}{ Gender } \\
\hline Male & $118(83.7)$ \\
\hline Female & $23(16.3)$ \\
\hline \multicolumn{2}{|l|}{ Smoking } \\
\hline No & $114(81.9)$ \\
\hline Yes & $27(19.1)$ \\
\hline \multicolumn{2}{|l|}{ Drinking } \\
\hline No & $114(80.9)$ \\
\hline Yes & $27(19.1)$ \\
\hline \multicolumn{2}{|l|}{ Family history of cancer } \\
\hline No & $103(73.0)$ \\
\hline Yes & 38 (27.0) \\
\hline \multicolumn{2}{|l|}{ Liver cirrhosis } \\
\hline No & $96(68.1)$ \\
\hline Yes & $45(31.9)$ \\
\hline \multicolumn{2}{|l|}{ Child-Pugh classification } \\
\hline A & $24(17.0)$ \\
\hline B & 69 (48.9) \\
\hline C & $48(34.1)$ \\
\hline \multicolumn{2}{|l|}{ TACE treatment } \\
\hline No & $71(50.4)$ \\
\hline Yes & 70 (49.6) \\
\hline \multicolumn{2}{|l|}{ TCM adjuvant treatment } \\
\hline No & $82(58.2)$ \\
\hline Yes & $59(41.8)$ \\
\hline \multicolumn{2}{|l|}{ Vital status } \\
\hline Dead & $88(62.4)$ \\
\hline Live & $53(37.6)$ \\
\hline Follow-up time, month (median, interquartile range) & $28.0(18.0-37.0)$ \\
\hline ccf-mtDNA content (median, interquartile range) & $1.98(1.17-3.68)$ \\
\hline
\end{tabular}


TABLE 2 | The association between ccf-mtDNA and overall survival of HBV-HCC patients.

\begin{tabular}{|c|c|c|c|c|c|c|c|c|}
\hline \multirow{2}{*}{$\begin{array}{l}\text { ccf- } \\
\text { mtDNA }\end{array}$} & \multirow{2}{*}{$\begin{array}{c}\text { Number of } \\
\text { patients }\end{array}$} & \multirow{2}{*}{$\begin{array}{c}\text { Number of } \\
\text { deaths }\end{array}$} & \multicolumn{2}{|c|}{ Univariate } & \multicolumn{2}{|l|}{ Multivariate $^{a}$} & \multicolumn{2}{|l|}{ Multivariate $^{\mathrm{b}}$} \\
\hline & & & HR (95\%Cl) & $P$-value & HR (95\%Cl) & $P$-value & HR $(95 \% \mathrm{Cl})$ & $P$ value \\
\hline \multicolumn{9}{|l|}{ By median } \\
\hline Lower & 71 & 45 & 1.00 & & 1.00 & & 1.00 & \\
\hline Higher & 70 & 43 & $\begin{array}{c}1.07 \\
(0.71-1.63)\end{array}$ & 0.740 & $\begin{array}{c}1.49 \\
(0.94-2.34)\end{array}$ & 0.089 & $\begin{array}{c}1.44 \\
(0.91-2.29)\end{array}$ & 0.120 \\
\hline \multicolumn{9}{|l|}{ By tertile } \\
\hline 1st tertile & 47 & 28 & 1.00 & & 1.00 & & 1.00 & \\
\hline 2nd tertile & 47 & 32 & $\begin{array}{c}1.45 \\
(0.87-2.41)\end{array}$ & 0.156 & $\begin{array}{c}1.58 \\
(0.89-2.80)\end{array}$ & 0.122 & $\begin{array}{c}1.60 \\
(0.90-2.84)\end{array}$ & 0.111 \\
\hline 3rd tertile & 47 & 28 & $\begin{array}{c}1.18 \\
(0.70-1.99)\end{array}$ & 0.545 & $\begin{array}{c}1.76 \\
(1.00-3.11)\end{array}$ & 0.051 & $\begin{array}{c}1.66 \\
(0.93-2.94)\end{array}$ & 0.084 \\
\hline$P$ for trend & & & & 0.364 & & 0.127 & & 0.156 \\
\hline
\end{tabular}

aMultivariate analysis adjusted for age, gender, drinking and smoking status, family history of cancer, liver cirrhosis, Child-Pugh classification, and TACE treatment.

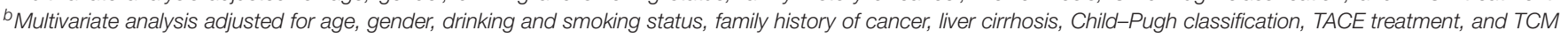
adjuvant treatment.

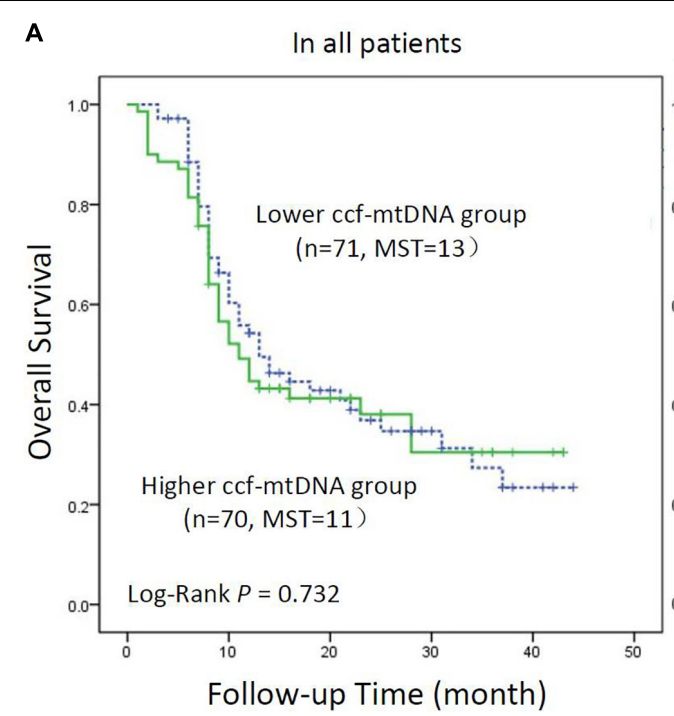

B In patients with TACE and TCM treatments

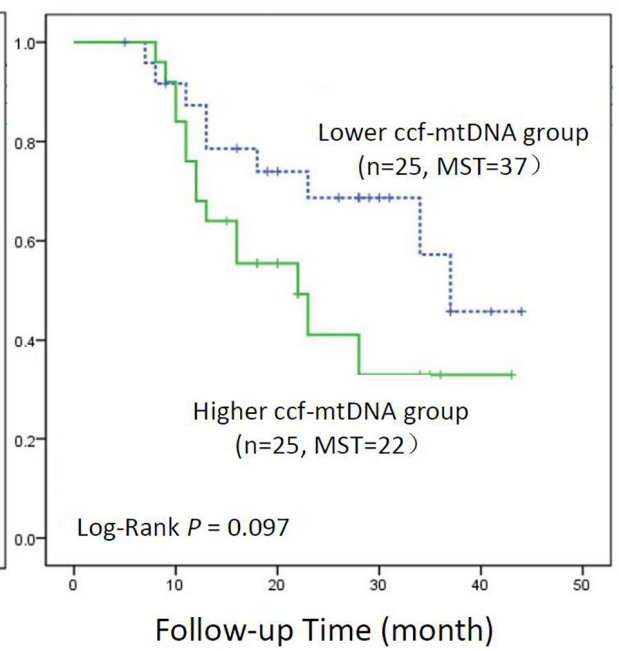

FIGURE 2 | Kaplan-Meier curve analysis of ccf-mtDNA content and hepatitis B virus-related hepatocellular carcinoma (HBV-HCC) patients. (A) All HBV-HCC patients $(n=141)$. (B) HBV-HCC patients receiving transarterial chemoembolization combined with traditional Chinese medicine patients $(n=50)$. MST indicates the median survival time.

history of family cancer, liver cirrhosis, Child-Pugh classification, and TACE treatment, a borderline significant association was observed. Compared to patients with low ccf-mtDNA, patients with high ccf-mtDNA content had an increased death risk with a hazard ratio (HR) of 1.49 (95\%CI, 0.94-2.34, $p=0.089)$ when a median value of ccf-mtDNA was used as the cutoff or with a HR of 1.76 (95\%CI, 1.00-3.11, $p=0.051$, the highest vs. the lowest tertile) when tertile values were used as the cutoffs. If further adjusting for TCM adjuvant treatment, the association remained borderline significant in the tertile analysis (HR, 1.66; 95\%CI, 0.93-2.94, $p=0.084$ ).

The Kaplan-Meier method was used to draw the survival curves of HBV-HCC patients with different ccf-mtDNA contents. As shown in Figure 2A, there was no statistically significant difference in the overall survival between patients with low and high ccf-mtDNA content $(\log \operatorname{rank} p=0.732)$.

\section{Association Between ccf-mtDNA Content and Overall Survival in HBV-HCC Patients Receiving TACE Combined With TCM}

We further evaluated whether ccf-mtDNA content was associated with overall survival in a subcohort of patients receiving TACE combined with TCM. The epidemiological and clinical characteristics of $50 \mathrm{HBV}-\mathrm{HCC}$ patients in this subcohort are summarized in Table 3. The average age was $56.48 \pm 8.18$ years. Similar to the overall population, 
TABLE 3 | Characteristics of the HBV-HCC subcohort with TACE and TCM treatments.

\begin{tabular}{|c|c|}
\hline Characteristics & Number $(n=50, \%)$ \\
\hline \multicolumn{2}{|l|}{ Age (median) } \\
\hline$\leq 55$ & $25(50.0)$ \\
\hline$>55$ & $25(50.0)$ \\
\hline \multicolumn{2}{|l|}{ Gender } \\
\hline Male & $43(86.0)$ \\
\hline Female & $7(14.0)$ \\
\hline \multicolumn{2}{|l|}{ Smoking status } \\
\hline No & $35(70.0)$ \\
\hline Yes & $15(30.0)$ \\
\hline \multicolumn{2}{|l|}{ Drinking status } \\
\hline No & $35(70.0)$ \\
\hline Yes & $15(30.0)$ \\
\hline \multicolumn{2}{|l|}{ Family history of cancer } \\
\hline No & $33(66.0)$ \\
\hline Yes & $17(34.0)$ \\
\hline \multicolumn{2}{|l|}{ Liver cirrhosis } \\
\hline No & $34(68.0)$ \\
\hline Yes & $16(32.0)$ \\
\hline \multicolumn{2}{|l|}{ Child-Pugh classification } \\
\hline A & $14(28.0)$ \\
\hline B & $26(52.0)$ \\
\hline C & $10(20.0)$ \\
\hline \multicolumn{2}{|l|}{ Tumor number } \\
\hline Single & $32(64.0)$ \\
\hline Multiple & $18(36.0)$ \\
\hline \multicolumn{2}{|l|}{ Tumor size } \\
\hline$\leq 5$ & $26(52.0)$ \\
\hline$>5$ & $24(48.0)$ \\
\hline \multicolumn{2}{|l|}{ Vital status } \\
\hline Death & $23(46.0)$ \\
\hline Live & $27(54.0)$ \\
\hline Follow-up time (month) (median, interquartile range) & $29.0(20.0-37.0)$ \\
\hline ccf-mtDNA content (median, interquartile range) & $1.58(0.77-3.27)$ \\
\hline
\end{tabular}

most of the patients were male (86\%), non-smokers (70\%), non-drinkers $(70 \%)$, and without a family history of cancer (73\%). There were $34(68 \%)$ patients who developed liver cirrhosis and 36 (72\%) patients with a B or C ChildPugh class. Twenty-three (46\%) patients died during a median follow-up of 29.0 months (interquartile range, 20.0-37.0 months).

The patients were then stratified into different risk groups by the median or tertile values of ccf-mtDNA content. The results from multivariate analyses showed that the high content of ccf-mtDNA was an independent prognostic factor for HBVHCC patients treated with both TACE and TCM (Table 4). A significantly higher death risk was observed in patients with high ccf-mtDNA content than in those with low ccf-mtDNA content (median cutoff: $\mathrm{HR}=4.09, p=0.018$; tertile cutoff: HR 4.89, $p=0.021$ in the middle tertile group, and HR 5.26, $p=0.039$ in the highest tertile group) after adjusting for age, gender, smoking and drinking status, family history of cancer, liver cirrhosis, Child-Pugh classification, tumor number, and tumor size.

Kaplan-Meier analysis was used to assess the survival difference in HBV-HCC patients with different levels of ccfmtDNA (Figure 2B). The result showed that the survival of patients with a high content of ccf-mtDNA was worse, but the difference in overall survival between the two groups did not reach statistical significance $(\log \operatorname{rank} p=0.097)$.

\section{DISCUSSION}

In this retrospective study, we measured the ccf-mtDNA content in HBV-HCC patients and demonstrated that patients with low ccf-mtDNA content had a survival benefit from TACE combined with TCM treatment. Moreover, the association between ccf-mtDNA and overall survival was independent of clinical confounders. A previous study showed that a low ccf-mtDNA content was associated with an increased risk for developing HBV-HCC (Li et al., 2016), while the current study suggested that the high level of ccf-mtDNA was associated with the poor outcome of HBV-HCC patients receiving TACE combined with TCM. The inconsistent findings may be explained by the different characteristics of the study populations, for example,

TABLE 4 | The association between ccf-mtDNA and overall survival of HBV-HCC patients with TACE and TCM treatments.

\begin{tabular}{|c|c|c|c|c|c|c|}
\hline \multirow[t]{2}{*}{ ccf-mtDNA } & \multirow{2}{*}{$\begin{array}{l}\text { Number of } \\
\text { patients }\end{array}$} & \multirow[t]{2}{*}{ Number of deaths } & \multicolumn{2}{|c|}{ Univariate } & \multicolumn{2}{|c|}{ Multivariate $^{\mathrm{a}}$} \\
\hline & & & HR $(95 \% \mathrm{Cl})$ & $P$-value & HR $(95 \% \mathrm{Cl})$ & $P$-value \\
\hline \multicolumn{7}{|l|}{ By median } \\
\hline Lower & 25 & 9 & 1.00 & & 1.00 & \\
\hline Higher & 25 & 14 & $2.02(0.86-4.74)$ & 0.097 & $4.09(1.27-13.17)$ & 0.018 \\
\hline 2nd tertile & 17 & 10 & $2.66(0.94-7.52)$ & 0.066 & $4.89(1.27-18.85)$ & 0.021 \\
\hline 3rd tertile & 16 & 7 & $1.60(0.53-4.85)$ & 0.403 & $5.26(1.09-25.31)$ & 0.039 \\
\hline$P$ for trend & & & & 0.173 & & 0.038 \\
\hline
\end{tabular}

a Multivariate analysis adjusted for age, gender, drinking and smoking status, family history of cancer, liver cirrhosis, Child-Pugh classification, tumor number and tumor size. 
ethnicity, tumor stage, liver function, and the treatments that the patients received.

ccf-mtDNA is the mtDNA fragments that are released outside the cell and into the circulation by cell necrosis and secretion. In the last decade, ccf-mtDNA has been demonstrated to be a potential non-invasive biomarker in different types of disease, including cancers. Several studies (Ellinger et al., 2012; Mahmoud et al., 2015; Li et al., 2016) showed that ccf-mtDNA in plasma or serum could be used as a diagnostic and prognostic marker in many solid tumors. The research of Ellinger showed that the ccf-mtDNA content in the serum of patients with urinary system malignancies increased significantly (Ellinger et al., 2012), and the study of Mahmoud showed that ccf-mtDNA had a prognostic value in breast cancer (Mahmoud et al., 2015). Meng et al. (2019) reported that increasing levels of ccf-mtDNA had a significant association with epithelial ovarian cancer progress and poor survival. These studies suggested that ccf-mtDNA is a potential tumor molecular marker, and our study further verified that ccf-mtDNA content in serum samples could be a potential non-invasive prognostic biomarker for HCC patients.

Transarterial chemoembolization is the first line of treatment for patients with intermediate stage of disease, including asymptomatic patients with limited unresectable multinodular lesions, without vascular invasion or extrahepatic spread, and who have well-preserved liver function (Raoul et al., 2019), although its clinical benefit is still far from satisfactory. A metaanalysis showed that TCM treatment improves the immune response for unresectable HCC after TACE treatment (Meng et al., 2011). TCM is gathering increasing interest due to the immunoregulatory properties of certain compounds which can restore immunosurveillance in HCC to promote antitumor effects in several pathways, including the upregulation of immunostimulatory factors and the downregulation of immunosuppressive factors (Jia and Wang, 2020). Several clinical studies have demonstrated that the utility of TCM can reduce adverse events after the TACE treatment of HCC patients. Tang et al. (2016) found that the Chinese medicine Jianpi Ligan decoction was effective in in reducing side effects and improving the long-term survival of patients with unresectable HCC treated by TACE. However, the cellular and molecular mechanisms of TCM-mediated anti-tumor effect are still not clear, and effective predictive and prognostic biomarkers are needed to increase the efficacy and improve the survival of HCC patients receiving treatment in the combination of TACE and TCM. In addition, mtDNA is also a well-known immune stimulator in many diseases including HCC. These previous findings may partially explain why, in this study, mtDNA content was associated with improved survival only in HCC patients who received both TACE and TCM, but not in the overall patient cohort. In our study, we also found that a high content of ccf-mtDNA was an

\section{REFERENCES}

Bao, D., Ba, Y., Zhou, F., Zhao, J., Ge, N., Yang, Q., et al. (2016). Alterations of telomere length and mtDNA copy number are associated with overall survival in hepatocellular carcinoma patients treated with transarterial independent prognostic factor for HBV-HCC patients receiving TACE combined with TCM treatment, suggesting its potential as a biomarker for identifying HCC patients who may benefit from this combination therapy.

There are several limitations in our study, for example, the small sample size $(n=50)$ in the subcohort may lead to an unstable estimation of the association between ccfmtDNA content and clinical outcome of HBV-HCC patients receiving TACE combined with TCM treatment. In addition, the lack of pathological tumor stage in the population may not comprehensively address the confounding effects in the multivariate analysis. Therefore, future studies with a large sample size and comprehensive clinical parameters are needed to verify these findings.

In summary, this study showed that the ccf-mtDNA content in serum had a prognostic value for HBV-HCC patients receiving TACE combined with TCM treatment and may be used as a potential biomarker for the outcome prediction of HCC patients.

\section{DATA AVAILABILITY STATEMENT}

The raw data supporting the conclusions of this article will be made available by the authors, without undue reservation.

\section{ETHICS STATEMENT}

The studies involving human participants were reviewed and approved by The Affiliated Fifth People's Hospital of Ganzhou in Gannan Medical University. The patients/participants provided their written informed consent to participate in this study.

\section{AUTHOR CONTRIBUTIONS}

SW and GZ conceived and designed the experiment and analyzed the data. GZ and JX wrote the manuscript. YL, SL, and HL performed the experiments. FX, XL, and QZ collected the samples and clinical data. All authors are in agreement with the content of the manuscript and this submission.

\section{FUNDING}

This work was supported by the National Natural Science Foundation of China (Grant No. 82060524), Science and Technology Innovation Outstanding Young Talents Training Program of Jiangxi Province (Grant No. 20192BCBL23017), and The Youth Jinggang Scholars Program in Jiangxi Province.

chemoembolization. Cancer Chemother. Pharmacol. 78, 791-799. doi: 10.1007/ s00280-016-3128-1

Chen, Q., Wu, P., Huang, T., Shen, L., and Huang, Z. (2019). Efficacy of treatment regimens for advanced hepatocellular carcinoma. Medicine 98:e17460. doi: 10. 1097/MD.0000000000017460 
Dilip, A., Cheng, G., Joseph, J., Kunnimalaiyaan, S., and Kalyanaraman, B. (2013). Mitochondria-targeted antioxidant and glycolysis inhibition: synergistic therapy in hepatocellular carcinoma. Anticancer Drugs 24, 881-888. doi: 10. 1097/CAD.0b013e32836442c6

Ellinger, J., Muller, D. C., Muller, S. C., Hauser, S., Bastian, P. J., Heukamp, L. C., et al. (2012). Circulating mitochondrial DNA in serum: a universal diagnostic biomarker for patients with urological malignancies. Urol. Oncol. 30, 509-515. doi: 10.1016/j.urolonc.2010.03.004

Fliss, M. S., Usadel, H., Caballero, O. L., Wu, L., and Buta, M. R. (2000). Facile detection of mitochondrial DNA mutations in tumors and bodily fluids. Science 287, 2017-2019. doi: 10.1126/science.287.5460.2017

Fu, X., Wan, S., Hann, H. W., Myers, R. E., and Hann, R. S. (2012). Relative telomere length: A novel a non-invasive biomarker for the risk of non-cirrhotic hepatocellular carcinoma in patients with chronic hepatitis B infection. Eur. J. Cancer 48, 1014-1022. doi: 10.1016/j.ejca.2012.02.066

Gomaa, A. I. (2015). Recent advances in multidisciplinary management of hepatocellular carcinoma. World J. Hepatol. 7:673. doi: 10.4254/wjh.v7.i4.673

Jia, W., and Wang, L. (2020). Using traditional chinese medicine to treat hepatocellular carcinoma by targeting tumor immunity. Evid. Based Complement Altern. Med. 2020:9843486. doi: 10.1155/2020/9843486

Li, L., Hann, H., Wan, S., Hann, R. S., and Wang, C. (2016). Cell-free circulating mitochondrial DNA content and risk of hepatocellular carcinoma in patients with chronic HBV infection. Sci. Rep. 6:23992. doi: 10.1038/srep23992

Mahmoud, E. H., Fawzy, A., Ahmad, O. K., and Ali, A. M. (2015). Plasma circulating cell-free nuclear and mitochondrial DNA as potential biomarkers in the peripheral blood of breast cancer patients. Asian Pac. J. Cancer Prev. 16, 8299-8305. doi: 10.7314/apjcp.2015.16.18.8299

Meng, M., Wen, Q., Cui, Y., She, B., and Zhang, R. (2011). Metaanalysis: Traditional chinese medicine for improving immune response in patients with unresectable hepatocellular carcinoma after transcatheter arterial chemoembolization. Explore 7, 37-43. doi: 10.1016/j.explore.2010.10.002

Meng, X., Schwarzenbach, H., Yang, Y., Müller, V., and Li, N. (2019). Circulating mitochondrial DNA is linked to progression and prognosis of epithelial ovarian cancer. Transl. Oncol. 12, 1213-1220. doi: 10.1016/j.tranon.2019.05.015

Raoul, J., Forner, A., Bolondi, L., Cheung, T. T., and Kloeckner, R. (2019). Updated use of TACE for hepatocellular carcinoma treatment: how and when to use it based on clinical evidence. Cancer Treat Rev. 72, 28-36. doi: 10.1016/j.ctrv.2018. 11.002

Reznik, E., Miller, M. L., Senbabaoglu, Y., Riaz, N., Sander, C., Sarungbam, J., et al. (2016). Mitochondrial DNA copy number variation across human cancers. Elife 5:10769. doi: 10.7554/eLife.10769

Tang, C. W., Zhu, M., Feng, W. M., Bao, Y., and Zheng, Y. Y. (2016). Chinese herbal medicine, Jianpi Ligan decoction, improves prognosis of unresectable hepatocellular carcinoma after transarterial chemoembolization: a retrospective study. Drug Des. Devel. Ther. 10, 2461-2466. doi: 10.2147/DDDT.S113295

Veltri, K. L., Espiritu, M., and Singh, G. (1990). Distinct genomic copy number in mitochondria of different mammalian organs. J. Cell Physiol. 143, 160-164. doi: $10.1002 /$ jcp.1041430122

Wan, S., Hann, H. W., Myers, R. E., Fu, X., and Hann, R. S. (2012). Telomere length in circulating serum DNA as a novel a non-invasive biomarker for cirrhosis: a nested case-control analysis. Liver Int. 32, 1233-1241. doi: 10.1111/j.1478-3231. 2012.02801.x

Weerts, M., Timmermans, E. C., van de Stolpe, A., Vossen, R., and Anvar, S. Y. (2018). Tumor-Specific mitochondrial DNA variants are rarely detected in Cell-Free DNA. Neoplasia 20, 687-696. doi: 10.1016/j.neo.2018. 05.003

Wenceslau, C. F., McCarthy, C. G., Szasz, T., Spitler, K., Szasz, T., Goulopoulou, S., et al. (2014). Mitochondrial damage-associated molecular patterns and vascular function. Eur. Heart J. 35, 1172-1177. doi: 10.1093/eurheartj/ehu047

Xie, Y. (2017). Hepatitis b Virus-Associated hepatocellular carcinoma. Adv. Exp. Med. Biol. 1018, 11-21. doi: 10.1007/978-981-10-5765-6_2

Xiong, Y., Lu, Q. J., Zhao, J., and Wu, G. Y. (2012). Metformin inhibits growth of hepatocellular carcinoma cells by inducing apoptosis via mitochondrionmediated pathway. Asian Pac. J. Cancer Prev. 13, 3275-3279. doi: 10.7314/apjcp. 2012.13.7.3275

Yu, M. (2012). Circulating cell-free mitochondrial DNA as a novel cancer biomarker: opportunities and challenges. Mitochondrial DNA 23, 329-332. doi: $10.3109 / 19401736.2012 .696625$

Conflict of Interest: The authors declare that the research was conducted in the absence of any commercial or financial relationships that could be construed as a potential conflict of interest.

Publisher's Note: All claims expressed in this article are solely those of the authors and do not necessarily represent those of their affiliated organizations, or those of the publisher, the editors and the reviewers. Any product that may be evaluated in this article, or claim that may be made by its manufacturer, is not guaranteed or endorsed by the publisher.

Copyright (c) 2021 Zhou, Li, Li, Liu, Xu, Lai, Zhang, Xu and Wan. This is an open-access article distributed under the terms of the Creative Commons Attribution License (CC BY). The use, distribution or reproduction in other forums is permitted, provided the original author(s) and the copyright owner(s) are credited and that the original publication in this journal is cited, in accordance with accepted academic practice. No use, distribution or reproduction is permitted which does not comply with these terms. 